\title{
Quiste de duplicación duodenal, diagnóstico prenatal y exéresis postnatal*
}

\author{
Drs. YOLANDA MARTÍNEZ-CRIADO', ANA MILLÁN L. ${ }^{1}$, \\ REYES VÁZQUEZ R., JUAN C. DE AGUSTÍN A. ${ }^{1}$ \\ 1 Servicio de Cirugía Pediátrica. Hospital Virgen del Rocío. \\ Sevilla, España.
}

Abstract

\section{Duodenal duplication cyst, prenatal diagnosis and postnatal excision}

Introduction: Duodenal duplication cysts are rare congenital anomalies, usually originate in the 2 nd -3 rd portion of the duodenum. The prenatal echography diagnosis shows a cyst in the right hypochondrium, and we should perform a differential diagnosis with other pathologies. Prenatal diagnosis will allow a correct and precocious postnatal management. Elective excision is the treatment of election preventing the development of complications. Clinical case: We present a rare case of duplication of the first portion of the duodenum that required precocious excision because obstruction to gastric emptying.

Key words: Duodenal duplication, prenatal diagnosis, obstruction to gastric emptying.

\section{Resumen}

Introducción: Los quistes de duplicidad duodenal son anomalías congénitas raras, que generalmente se originan en la $2^{\mathrm{a}}-3^{\mathrm{a}}$ porción duodenal. Ante el hallazgo en la ecografía prenatal de un quiste en hipocondrio derecho se debe realizar un diagnóstico diferencial con otras patologías. El diagnóstico prenatal, permitirá un correcto y precoz manejo postnatal. La exéresis electiva es el tratamiento de elección evitando la aparición de complicaciones posteriores. Caso clínico: Presentamos un caso excepcional de duplicidad de la primera porción duodenal, que precisó de exéresis precoz por causar obstrucción al vaciamiento gástrico.

Palabras clave: Duplicidad duodenal, diagnóstico prenatal, obstrucción al vaciamiento gástrico.

\section{Introducción}

Los quiste de duplicación duodenal, son malformaciones congénitas raras y con una gran variedad anatómica. Generalmente, se originan de la segunda y tercera posición duodenal, no existiendo ningún caso descrito en la literatura de duplicidad de la primera porción. La localización y tamaño del quiste, condiciona la sintomatología del paciente ${ }^{1}$.

El diagnóstico ecográfico prenatal permite realizar un seguimiento durante todo el embarazo y un correcto manejo postnatal. La tomografía computa-

*Recibido el 17 de diciembre de 2012 y aceptado para publicación el 31 de enero de 2013.

No existen conflictos de interés ni financiación.

Correspondencia:

Dra. Yolanda Martínez Criado

C/ Manuel Siurot, s/n. Sevilla, España.

yoli84mc@hotmail.com 

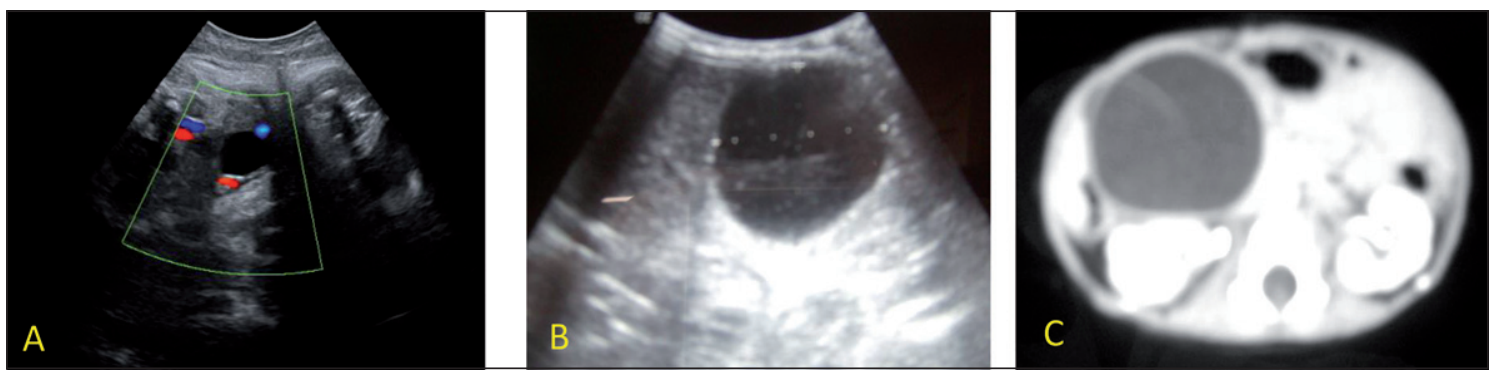

Figura 1. A) Ecografía prenatal a las 20 semanas. Hallazgo del quiste en hipocondrío derecho. B) Ecografía postnatal. C) TC postnatal, quiste de duplicación de $4 \mathrm{~cm}$.
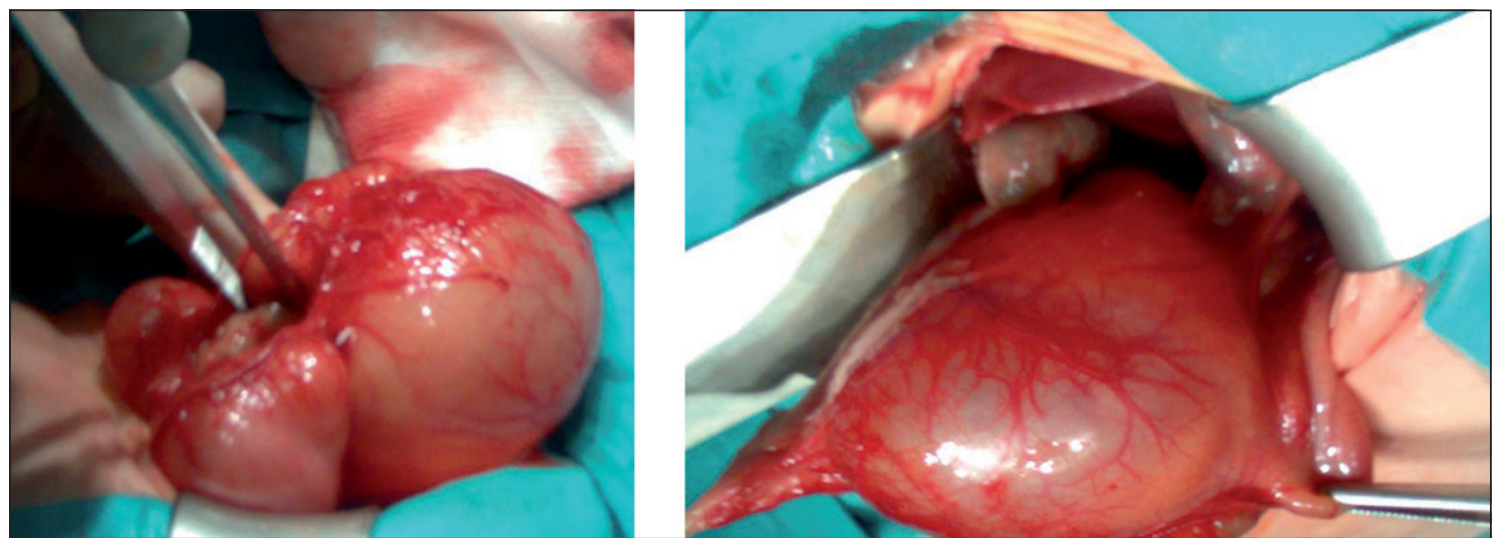

Figura 2. Quiste de duplicación de primera porción duodenal, que comprime el píloro.

rizada (TC) al nacimiento, nos ayudará a confirmar el diagnóstico y diferenciarlo de quistes de origen distinto: ovárico, renal, hepático, colédoco, mesentérico ${ }^{2}$.

El tratamiento de elección en pacientes asintomáticos es la cirugía electiva precoz antes del desarrollo de complicaciones. A continuación presentamos un caso de quiste de duplicidad de la primera porción duodenal que originó obstrucción al vaciamiento gástrico, precisando de cirugía urgente en los primeros días de vida.

\section{Caso clínico}

En la ecografía prenatal de la semana 20 de gestación de una primípara de 30 años, se objetivó la presencia de un quiste de 2 centímetros en hipocondrio derecho (Figura 1A). El seguimiento ecográfico durante el embarazo mostró un aumento de 3 a 4 centímetros del quiste. El parto fue espontáneo vaginal a las 40 semanas, con un apgar 10/10/10 y un peso de $3.690 \mathrm{~g}$. No presentaba anomalías asociadas.

Al nacimiento en el control ecográfico se seguía visualizando el quiste (Figura 1B), sin poder deter- minar la naturaleza del mismo. Se realizó un TC (Figura 1C) en el que se objetiva un quiste de $4 \mathrm{~cm}$ adyacente a duodeno, antro y páncreas.

$\mathrm{Al} 4^{\circ}$ día de vida, precisó de intervención quirúrgica urgente al comenzar con intolerancia enteral. Se realizó laparotomía transversa supraumbilical derecha, hallándose un quiste de $4 \mathrm{~cm}$, de duplicación de la primera porción duodenal que generaba una compresión extrínseca del píloro causando obstrucción al vaciamiento gástrico (Figura 2). Se realizó exéresis de la mitad anterior del quiste y mucosectomía de la pared común con el duodeno, añadiéndose piloroplastía.

La evolución postoperatoria fue favorable, recibiendo el alta al décimo día de vida.

El estudio histopatológico del quiste objetivó la presencia de mucosa gástrica ectópica.

\section{Discusión}

Los quistes de duplicación intestinal fueron descritos por Fitz (1884) y Ladd (1937). Estas duplicaciones comparten vascularización y en ocasiones pared muscular común con el tracto gastrointestinal. La mayoría de las duplicaciones entéricas son 
yeyuno-ileales (53\%), seguidas de mediastínicas y colónicas. Tan sólo un 5\% son duplicaciones duodenales, siendo excepcional la localización en la primera porción duodenal ${ }^{1,2}$.

Ante el hallazgo ecográfico prenatal de quiste en hipocondrio derecho, se debe de realizar un diagnóstico diferencial con otras patologías: quiste ovárico, quiste renal, quiste hepático o de colédoco, quiste mesentérico o quiste de duplicación intestinal. La presencia ecográfica del signo de "doble pared" y la peristalsis del quiste, son datos sugestivos de duplicación intestinal. Un tercio de los pacientes asocia otras anomalías, por lo que la resonancia magnética prenatal, podría ser útil para la caracterización del quiste y descartar patologías concomitantes ${ }^{2}$. El seguimiento ecográfico prenatal seriado, permite detectar complicaciones o crecimiento excesivo, que en ocasiones precisa de un tratamiento intrauterino. Al nacimiento, el TC es la prueba complementaria de elección que nos permite realizar el diagnóstico diferencial con otras patologías, así como determinar las características o posibles complicaciones ${ }^{3}$.

La sintomatología de los quistes de duplicación intestinal es muy variable dependiendo del tipo y localización. Normalmente permanecen asintomáticos, pero en ocasiones el desarrollo de complicaciones hace necesario una cirugía en los primeros días de vida, como es el caso que hemos presentado. Las potenciales complicaciones de estos quistes pueden ser muchas y muy severas. El dolor abdominal es la manifestación precoz más frecuente, generado por un aumento de la presión intraquística al acumularse secreciones. En ocasiones causan obstrucción intestinal por compresión extrínseca, actúan como cabeza de invaginación o desencadenan vólvulos. La presencia de tejido gástrico ectópico, presente hasta en un $20-30 \%$ de los quistes, puede provocar ulceración, sangrado o perforación. Finalmente, se pueden generar cambios malignos más a largo plazo $^{4,5}$.

Existe controversia en cuál es el momento óptimo para realizar la cirugía. El diagnóstico ecográfico prenatal, y su confirmación con una tomografía computarizada al nacimiento, permite planificar cirugía electiva precoz, generalmente antes de los 6 meses para evitar el desarrollo de posibles complicaciones. La necesidad de cirugía en los primeros días de vida por complicaciones es infrecuente, pero en nuestro caso, el tamaño del quiste y su inusual localización en la primera porción duodenal, generó una obstrucción al vaciamiento gástrico que requirió una exéresis urgente ${ }^{6}$.

Respecto al tipo de abordaje quirúrgico, laparoscópico versus laparotómico, no existe evidencia científica de cuál es el de elección. Aunque la tendencia actual es a realizar un abordaje laparoscópico, que provoca menos dolor y mejor resultado estético. En nuestro caso, la laparotomía se consideró más segura al tratarse de un neonato, con un quiste de gran tamaño?

\section{Referencias}

1. Merrot T, Anastasescu R, Pankevych T, Tercier S, Garcia S, Alessandrini P, et al. Duodenal duplications. Clinical characteristics, embryological hypotheses, histological findings, treatment. Eur J Pediatr Surg. 2006;16:18-23.

2. Borgnon J, Durand C, Gourlaouen D, Sagot P, Sapin E. Antenatal detection of a communicating duodenal duplication. Eur J Pediatr Surg. 2003;13:130-3.

3. Laje P, Flake AW, Adzick NS. Prenatal diagnosis and postnatal resection of intraabdominal enteric duplications. J Pediatr Surg. 2010;45:1554-8.

4. Gonzálvez Piñera J, Fernández Córdoba MS, Hernández Anselmi E. Acute abdomen caused by infected duodenal duplication. Cir Pediatr. 2008;21:181-4.

5. Kawahara H, Takahashi T, Okada A. Characteristics of duodenal duplications causing pancreatitis in children and adolescents: a case report and review of the literature. J Pediatr Gastroenterol Nutr. 2002;35:372-6.

6. Foley PT, Sithasanan N, McEwing R, Lipsett J, Ford WD, Furness M. Enteric duplications presenting as antenatally detected abdominal cysts: is delayed resection appropriate?. J Pediatr Surg. 2003;38:1810-3.

7. Holcomb GW 3rd, Gheissari A, O’Neill JA Jr, Shorter NA, Bishop HC. Surgical management of alimentary tract duplications. Ann Surg. 1989;209:167-74. 